\title{
EXPONENTIAL MONOMIALS ON HYPERGROUP JOINS
}

\author{
KEDUMETSE VATI AND LÁSZLÓ SZÉKELYHIDI \\ Dedicated to the memory of Prof. Herbert Heyer
}

Received 13 August, 2019

\begin{abstract}
Exponential monomials and polynomials are the basic building blocks of spectral synthesis. Recently a systematic study of exponential polynomials has been started on hypergroups. In this paper we join these investigations and describe exponential polynomials on hypergroup joins.
\end{abstract}

2010 Mathematics Subject Classification: 20N20; 39B99

Keywords: hypergroup join, functional equation

\section{INTRODUCTION}

We started the study of basic function classes on hypergroup joins in our paper [14]. The basic function classes we are studying play a fundamental role in spectral analysis and spectral synthesis (see [9,12]). In this paper we continue this work and describe further basic function classes, called moment functions on hypergroup joins (see [1]). In the sequel $\mathbb{C}$ denotes the set of complex numbers. By a hypergroup we always mean a locally compact hypergroup. For basics about hypergroups see the monograph [1]. A comprehensive monograph on functional equations on hypergroups is [7].

Let $K$ be a hypergroup with identity $e$ and involution ${ }^{\vee}$. The non-identically zero continuous function $m$ is called an exponential on $K$ if $m: K \rightarrow \mathbb{C}$ satisfies $m(x * y)=m(x) m(y)$ for each $x, y$ in $K$. The description of exponentials on some types of commutative hypergroups can be found in [7]. In [11] (see also [10]) the author defined the concept of exponential monomial on commutative hypergroups: let $K$ be a commutative hypergroup and $f: K \rightarrow \mathbb{C}$ a continuous function. We say that $f$ is a generalized exponential monomial, if there exists an exponential $m: K \rightarrow \mathbb{C}$ and a natural number $n$ such that

$$
\Delta_{m ; y_{1}, y_{2}, \ldots, y_{n+1}} * f=0
$$

László Székelyhidi was supported by the Hungarian National Foundation for Scientific Research (OTKA), Grant No. K111651 and by BIUST, Botswana. 
holds for every $y_{1}, y_{2}, \ldots, y_{n+1}$ in $K$. We recall that $\Delta_{m ; y_{1}, y_{2}, \ldots, y_{n+1}}$ is the convolution product

$$
\Delta_{m ; y_{1}, y_{2}, \ldots, y_{n+1}}=\Pi_{k=1}^{n+1}\left(\boldsymbol{\delta}_{\breve{y}}-m(y) \boldsymbol{\delta}_{o}\right),
$$

where, in general, $\delta_{x}$ denotes the point mass supported at $x$ in $K$, and $o$ denotes the identity in $K$. It is known that if $f$ is nonzero, then the exponential $m$ is unique, and the smallest $n$ with the above property is called the degree of $f$. In this case we say that $f$ is a generalized m-exponential monomial, or $f$ is associated with $\mathrm{m}$. We call $f$ simply an exponential monomial, if the linear space generated by all translates $\delta_{\breve{y}} * f$ is finite dimensional. Clearly, any constant multiple of the exponential $m$ is an $m$ exponential monomial, and if the constant is nonzero, then it has degree 0 . Another simple example is that of the $m$-sine functions: $f$ is called an $m$-sine function, if it satisfies

$$
f(x * y)=f(x) m(y)+f(y) m(x)
$$

for each $x, y$ in $K$. If $f$ is nonzero, then it is an $m$-exponential monomial of degree 1 . Linear combinations of generalized exponential monomials are called generalized exponential polynomials, and linear combinations of exponential monomials are called exponential polynomials. We note that quadratic functions (see [14]) are not necessarily exponential polynomials, but if the hypergroup structure on $K$ arises from a group structure, then every quadratic function is a generalized exponential monomial associated with the exponential identically 1 .

Exponential polynomials have fundamental importance in spectral analysis and synthesis. Spectral synthesis on hypergroups has been investigated in the works $[7,9]$. For more about spectral analysis and spectral synthesis see the monograph [12]. Characterization of these functions classes and related functional equations on different types of hypergroups have been studied in several papers(see e.g $[2,8])$. In this paper we describe generalized exponential classes on hypergroup joins. The hypergroup join construction is a special way to unite two hypergroups, one of them is compact and the other is discrete. Hypergroup joins and their duals have been studied in [17]. Hypergroup joins were generalized by M. Voit [15] and by H. Heyer and S. Kawakami [3], based on exact sequences.

Another important class is presented by the class of moment functions. According to the terminology in [7] we say that the continuous functions $f_{n}: K \rightarrow \mathbb{C}$ ( $n=0,1, \ldots, N, N$ is a natural number) on the hypergroup $K$ form a (generalized) moment function sequence of order $N$ if $f_{0}$ is non-identically zero and

$$
f_{n}(x * y)=\sum_{k=0}^{n}\left(\begin{array}{l}
n \\
k
\end{array}\right) f_{k}(x) f_{n-k}(y)
$$

holds for each $x, y$ in $K$ and for $k=0,1, \ldots, N$ (see e.g [4-6]). Clearly, $f_{0}$ is an exponential and we say that the sequence $\left(f_{n}\right)_{n \in \mathbb{N}}$ is associated with the exponential $f_{0}$. For the sake of simplicity we omit the adjective "generalized". The function $f_{k}$ in this sequence is called a moment function of order $k$. Hence moment functions of 
order 0 are exactly the exponentials and moment functions of order 1 associated with the exponential $m=f_{0}$ are exactly the $m$-sine functions. In [13] generalized moment functions on hypergroup joins were characterized and described. We shall see below that generalized moment functions are exponential monomials.

\section{HYPERGROUP JOIN}

The definition of hypergroup join can be found in [1], p. 59. Here we recall the construction. Let $(C, *)$ be a compact hypergroup with normalized Haar measure $\omega_{C}$ and $(D, \cdot)$ a discrete hypergroup with $C \cap D=\{e\}$, the identity of both hypergroups. The hypergroup join $C \vee D$ is the set $C \cup D$ with the unique topology for which both $C$ and $D$ are closed subspaces. Involution on $C \cup D$ is defined in the way that its restriction to $C$ and to $D$, respectively, coincides with the involution on $C$ and on $D$, respectively. Convolution on $C \vee D$ is defined in the following way:

(1) For $x, y$ in $C$ the convolution of $\delta_{x}$ and $\delta_{y}$ is $\delta_{x} * \delta_{y}$.

(2) For $x, y$ in $D$ and $x \neq \breve{y}$ the convolution of $\delta_{x}$ and $\delta_{y}$ is $\delta_{x} \cdot \delta_{y}$.

(3) For $x$ in $C$ and $y \neq e$ in $D$ the convolution of $\delta_{x}$ and $\delta_{y}$ and also the convolution of $\delta_{y}$ and $\delta_{x}$ is $\delta_{y}$.

(4) For $y \neq e$ in $D$ we have the unique representation

$$
\delta_{y} \cdot \delta_{\breve{y}}=\sum_{w \in D} c_{w} \delta_{w}
$$

with some complex numbers $c_{w}$ for $w$ in $D$. Then the convolution of $\delta_{y}$ and $\delta_{\breve{y}}$ and also the convolution of $\delta_{\breve{y}}$ and $\delta_{y}$ is

$$
c_{e} \omega_{C}+\sum_{w \in D, w \neq e} c_{w} \delta_{w}=\delta_{y} \cdot \delta_{\breve{y}}+c_{e}\left(\omega_{C}-\delta_{e}\right) .
$$

For the sake of simplicity, by virtue of 1 . above, we denote the convolution in $C \vee D$ with $*$, too. We note that commutativity is not assumed in $C$ nor in $D$. In fact, $C \vee D$ is commutative if and only if $C$ and $D$ is commutative. Clearly, $C$ is a compact subhypergroup of $C \vee D, D$ is a discrete subset of $C \vee D$, but $D$ is not necessarily a subhypergroup of $C \vee D$. For further information about hypergroup joins and their applications see [1].

In what follows we shall always assume that $D \neq\{e\}$ and we denote $D \backslash\{e\}$ with $D_{e}$. It follows that $D_{e}$ is nonempty.

\section{MOMENT FUnCTIONS AS EXPONENTIAL MONOMIALS}

A special case of exponential monomials is presented by the generalized moment functions as it is shown in the following theorem.

Theorem 1. Let $K$ be a commutative hypergroup and $\left(\varphi_{n}\right)_{n \in \mathbb{N}}$ a generalized moment function sequence. Then $\varphi_{n}$ is an exponential monomial of degree at most $n$ for each $n$. 
Proof. By definition, the sequence satisfies

$$
\varphi_{n}(x * y)=\sum_{k=0}^{n} \varphi_{k}(x) \varphi_{n-k}(y)
$$

for each $x, y$ in $K(n=0,1, \ldots)$. We prove the statement by induction on $n$ and it is obvious for $n=0$. Clearly $m=\varphi_{0}$ is an exponential. Suppose that $n \geq 1$ and we have proved our statement for $k=0,1, \ldots, n-1$. Now we prove it for $k=n$. Let $y_{1}, y_{2}, \ldots, y_{n+1}$ be arbitrary in $K$. We have

$$
\begin{aligned}
& \Delta_{m ; y_{1}, y_{2}, \ldots, y_{n+1}} \varphi_{n}(x)=\Delta_{m ; y_{1}, y_{2}, \ldots, y_{n}}\left(\varphi_{n}\left(x * y_{n+1}\right)-m\left(y_{n+1}\right) \varphi_{n}(x)\right) \\
& =\Delta_{m ; y_{1}, y_{2}, \ldots, y_{n}}\left(\sum_{k=0}^{n}\left(\begin{array}{l}
n \\
k
\end{array}\right) \varphi_{k}(x) \varphi_{n-k}\left(y_{n+1}\right)\right)-m\left(y_{n+1}\right) \Delta_{m ; y_{1}, y_{2}, \ldots, y_{n}} \varphi_{n}(x) \\
& =\Delta_{m ; y_{1}, y_{2}, \ldots, y_{n}} \varphi_{n}(x) . m\left(y_{n+1}\right)-m\left(y_{n+1}\right) \Delta_{m ; y_{1}, y_{2}, \ldots, y_{n+1}} \varphi_{n}(x)=0,
\end{aligned}
$$

which proves the statement.

Theorem 2. Let $K$ be a commutative hypergroup and $\Phi: K \times \mathbb{C}^{n} \rightarrow \mathbb{C}$ an exponential family for $K$. Let $N$ be a nonnegative integer and $1 \leq i \leq n$ an integer. Then for every polynomial $P: \mathbb{C}^{n} \rightarrow \mathbb{C}$ of degree $N$ the function $x \mapsto P\left(\partial_{\lambda}\right) \Phi(x, \lambda)$ is an exponential monomial of degree at most $N$.

Proof. The proof can be found in [10, Theorem 3].

\section{EXPONENTIAL MONOMIALS ON COMPACT HYPERGROUPS}

Theorem 3. On a compact commutative hypergroup every nonzero generalized exponential monomial is of degree zero.

Proof. We prove the statement by induction on the degree of the generalized $m$ exponential monomial $f \neq 0$, and it is obvious if $\operatorname{deg} f=0$. First we note that every nonzero generalized $m$-exponential polynomial of degree 0 is a constant multiple of $m$. Indeed, we have

$$
0=\Delta_{m ; y} * f(x)=f(x * y)-m(y) f(x)
$$

for each $x, y$ in $K$. Interchanging $x$ and $y$ we obtain $f(x) m(y)=f(y) m(x)$, hence the substitution $y=o$ gives $f(x)=f(o) m(x)$.

We introduce the function $g(x)=f(x)-f(o) m(x)$. Then, by the obvious property

$$
\Delta_{m ; y} * g=\Delta_{m ; y} * f
$$

it follows that $g$ is a generalized $m$-exponential monomial of degree at most $n$. In addition, $g(o)=0$.

We assume that we have proved that every nonzero generalized $m$-exponential polynomial of degree at most $n-1$ is of degree zero, and now we prove it for $\operatorname{deg} g \leq n$. By assumption,

$$
\Delta_{m ; y_{1}, y_{2}, \ldots, y_{n}} *\left(\Delta_{m ; y} * g\right)(x)=\Delta_{m ; y, y_{1}, y_{2}, \ldots, y_{n}} * g(x)=0,
$$


that is, $\Delta_{m ; y} * g$ is a generalized $m$-exponential polynomial of degree at most $n-1$, hence $\Delta_{m ; y} * g=c(y) m(x)$ holds for each $y$ in $K$, where $c: K \rightarrow \mathbb{C}$ is some continuous function. In other words, we have

$$
g(x * y)=m(y) g(x)+c(y) m(x)
$$

for each $x, y$ in $K$. Putting $x=o$ we have $g=c$, hence $g$ is an $m$-sine function, and by the results in [16], $g$ is identically zero. It follows that $f=f(o) m$ and our theorem is proved.

In [16] M. Voit proved that on commutative compact hypergroups every $m$-sine function is zero. It is not known if this statement is true on non-commutative compact hypergroups. The following theorem shows that it is true at least for 1-sine functions, that is, for additive functions. In fact, we prove a stronger statement.

Theorem 4. On a compact hypergroup every generalized 1-exponential monomial is constant.

Proof. Let $f: K \rightarrow \mathbb{C}$ be a generalized 1-exponential monomial on the compact hypergroup $K$. If $\operatorname{deg} f=0$, then we have $f(x * y)-f(x)=0$, hence with $x=o$ it follows $f(y)=f(o)$ for each $y$ in $K$.

Assume that we have proved our statement for $\operatorname{deg} f \leq n-1$ and now we let $\operatorname{deg} f=n \geq 1$. We have for $y, y_{1}, y_{2}, \ldots, y_{n}$ in $K$

$$
0=\Delta_{1 ; y_{1}, y_{2}, \ldots, y_{n}, y} * f(x)=\Delta_{1 ; y_{1}, y_{2}, \ldots, y_{n}} *\left(\Delta_{1 ; y} * f\right)(x)=0,
$$

hence, by assumption, $\Delta_{1 ; y} * f$ is a constant:

$$
\Delta_{1 ; y} * f(x)=f(x * y)-f(x)=c(y)
$$

holds for each $x, y$ in $K$, where $c: K \rightarrow \mathbb{C}$ is a continuous function. We have

$$
f(x * y)=f(x)+c(y)
$$

whenever $x, y$ is in $K$. Using associativity we have

$$
f(x * y * z)=f(x * y)+c(z)=f(x)+c(y)+c(z),
$$

and

$$
f(x * y * z)=f(x)+c(y * z),
$$

hence we infer $c(y * z)=c(y)+c(z)$ for each $y, z$ in $K$. As $K$ is compact and $c$ is continuous, the range of $c$ is compact in $\mathbb{C}$. If there is an $x_{0}$ such that $c\left(x_{0}\right) \neq 0$, then $c\left(n \cdot x_{0}\right)=c\left(x_{0} * x_{0} * \ldots x_{0}\right)=n \cdot c\left(x_{0}\right)$, which implies that the range of $c$ is unbounded, a contradiction. Hence $c \equiv 0$ and $f$ is constant.

In [14] we proved that at least the integral of every $m$-sine function is zero on any compact hypergroup. Now we prove the analogous result for generalized $\mathrm{m}$ exponential functions on compact hypergroups. We note that, clearly, on a compact hypergroup the integral of a constant is the constant itself, as the integral always refers to the integral with respect to the unique normalized Haar measure. 
Theorem 5. On a compact hypergroup the integral of every non-constant generalized m-exponential monomial is zero.

Proof. Let $K$ be a compact hypergroup with normalized Haar measure $d x$. We can show easily, by induction on $n$, that for any continuous function $g: K \rightarrow \mathbb{C}$ and exponential $m$ on $K$ we have

$$
\int_{K} \Delta_{m ; y_{1}, y_{2}, \ldots, y_{n+1}} * g(x) d x=\Pi_{k=1}^{n+1}\left(1-m\left(y_{k}\right)\right) \int_{K} g(x) d x
$$

for each $y_{1}, y_{2}, \ldots, y_{n+1}$ in $K$. Indeed, th statement is true for $n=0$.

Clearly, this equation implies the statement. Indeed, if $g$ is a generalized $m$ exponential monomial of degree at most $n$, then the left side is zero for each $y_{1}, y_{2}, \ldots, y_{n+1}$ in $K$. Hence the right side is zero, too, consequently if $\int_{K} g(x) d x \neq 0$, then $m \equiv 1$, that is $g$ is a generalized 1-exponential monomial, and, by the previous theorem, it is constant, which proves our statement.

We note that generalized 1-exponential polynomials are called generalized polynomials.

\section{EXPONENTIAL MONOMIALS ON HYPERGROUP JOINS}

Here we recall the theorem about the description of exponentials on hypergroup joins (see [14, Theorem 1]).

Theorem 6. Let $C, D$ be as above. The continuous function $m: C \cup D \rightarrow \mathbb{C}$ is an exponential on the hypergroup join $C \vee D$ if and only if one of the following possibilities holds:

i) $\left.m\right|_{C} \neq 1$ is an exponential on $C$ and $\left.m\right|_{D_{e}}$ is identically zero;

ii) $\left.m\right|_{C}$ is identically 1 and $\left.m\right|_{D}$ is an exponential on $D$.

Our main theorem follows.

Theorem 7. Let $C$ be a compact hypergroup and $D$ a discrete commutative hypergroup. Then the continuous function $f: C \cup D \rightarrow \mathbb{C}$ is a generalized exponential monomial of degree at most $n$ on the hypergroup join $C \vee D$ if and only if any of the following cases holds:

i) $\left.f\right|_{C}$ is a generalized exponential monomial of degree at most $n$ associated with an exponential $m_{C} \neq 1$ on $C$, and $\left.f\right|_{D_{e}}$ is zero.

ii) $\left.f\right|_{C}$ is constant, and $\left.f\right|_{D}$ is a generalized exponential monomial of degree at most $n$ on $D$.

Proof. In this proof we shall denote the convolution on $C$ and on $C \vee D$ by $x * y$, and on $D$ by $x \cdot y$, further $\omega_{C}$ denotes the unique normalized Haar measure on the compact hypergroup $C$. We note that $\left.f\right|_{C}$ is always a generalized exponential monomial of degree at most $n$ on $C$, as the convolution on $C \vee D$ coincides with the convolution on $C$ and the restriction of any exponential on $C \vee D$ to $C$ is an exponential on $C$. 
Let $f$ be a generalized $m$-exponential monomial of degree at most $n$ on $C \vee D$. By the previous theorem, we have two possibilities for $m$. In the first case $\left.m\right|_{C}$ is an exponential on $C$, and $\left.m\right|_{D_{e}} \equiv 0$. Then clearly, $\left.f\right|_{C}$ is a generalized $\left.m\right|_{C}$-exponential monomial of degree at most $n$ on $C$. On the other hand, let $y \neq e$ be in $D$, then we have for each $x$ in $D_{e}$ :

$$
0=\Delta_{m ; y}^{n+1} * f(x)=f(x),
$$

hence $f$ is identically zero on $D_{e}$, which is case $i$ ) above.

In the second case $\left.m\right|_{C}$ is identically 1 , and $\left.m\right|_{D}$ is an exponential on $D$. Clearly, $\left.f\right|_{C}$ is a generalized 1-exponential monomial on $C$, hence it is constant, by Theorem 4. We claim that $\left.f\right|_{D}$ is a generalized $\left.m\right|_{D}$-exponential monomial of degree at most $n$ on $D$. We have to prove the equality

$$
\Delta_{\left.m\right|_{D} ; y_{1}, y_{2}, \ldots, y_{n+1}} \cdot f(x)=0
$$

whenever $x, y_{1}, y_{2}, \ldots, y_{n+1}$ are in $D$. Here $\Delta$ is formed using the convolution in $D$ which is the same as in $C \vee D$ if there is no pair among the elements $x, y_{1}, y_{2}, \ldots$, $y_{n+1}$ which are involutive to each other. But if there are such pairs $z, \breve{z}$, then we have

$$
f(z * \check{z})=f(z \cdot \check{z})+c_{e}\left(\int_{C} f(t) d \omega_{C}(t)-f(e)\right)=f(z \cdot \check{z}),
$$

as $\left.f\right|_{C}$ is constant, that is $\left.f\right|_{C}=f(e)$, which implies our statement.

For the converse we suppose first that $i$ ) holds, and $\left.f\right|_{C}$ is a generalized $m_{C^{-}}$ exponential monomial of degree at most $n$ on $C$, where $m_{C} \neq 1$ is an exponential on $C$, further $\left.f\right|_{D_{e}}$ is zero. First we note, that $\left.f\right|_{C}$ is non-constant, as $m_{C} \neq 1$. We define $m: C \cup D \rightarrow \mathbb{C}$ as $m(x)=m_{C}(x)$ for $x$ in $C$, and $m(x)=0$ for $x$ in $D_{e}$. Then, by Theorem 1 in [14], $m$ is an exponential on $C \vee D$. We show that $f$ is a generalized $m$-exponential monomial of degree at most $n$ on $K$.

If $x, y_{1}, y_{2}, \ldots, y_{n+1}$ are in $C$, then clearly we have

$$
\Delta_{m ; y_{1}, y_{2}, \ldots, y_{n+1}} * f(x)=\left.\Delta_{m_{C} ; y_{1}, y_{2}, \ldots, y_{n+1}} * f\right|_{C}(x)=0 .
$$

Let $y$ be in $D$ and $x$ in $C$. Then $f(x * y)$ is either $f(x)$ or $f(y)=0$ depending on if $y=e$ or $y \neq e$, by the definition of the convolution on $K$. Hence

$$
\Delta_{m ; y} * f(x)= \begin{cases}f(x)-f(x)=0 & \text { if } y=e \\ f(y)-m(y) f(y)=0 & \text { if } y \neq e .\end{cases}
$$

It follows that

$$
\Delta_{m ; y_{1}, y_{2}, \ldots, y_{n+1}} * f(x)=0
$$

if $x$ is in $C$ and at least one of the $y_{i}$ 's is in $D$.

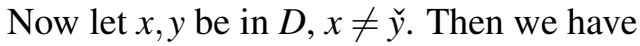

$$
\Delta_{m ; y} * f(x)=f(x * y)-m(y) f(x)= \begin{cases}f(x * y)=f(x \cdot y)=0 & \text { if } y \neq e \\ 0 & \text { if } y=e .\end{cases}
$$


The first part follows form the fact that if $x \neq \check{y}$, then $e$ is not in the support of $x \cdot y$, hence $f(x \cdot y)=\sum_{w \in D_{e}} c_{w} f(w)=0$, as $f$ vanishes on $D_{e}$. On the other hand, if $x=\check{y}$, then we have

$$
\Delta_{m ; y} * f(x)=f(\check{y} * y)-m(y) f(\check{y})= \begin{cases}f(\check{y} * y) & \text { if } y \neq e \\ 0 & \text { if } y=e .\end{cases}
$$

We recall that, by the definition of the convolution on $C \vee D$, we have

$$
\delta_{\check{y}} * \delta_{y}=\delta_{y} \cdot \delta_{\breve{y}}+c_{e}\left(\omega_{C}-\delta_{e}\right),
$$

where $c_{e}$ is the coefficient of $\delta_{e}$ in the expansion

$$
\delta_{y} \cdot \delta_{\breve{y}}=\sum_{w \in D} c_{w} \delta_{w}
$$

on the hypergroup $D$. It follows

$$
\begin{aligned}
f(\check{y} * y) & =f(\check{y} \cdot y)+c_{e}\left(\int_{C} f(t) d \omega_{C}(t)-f(e)\right) \\
& =\sum_{w \in D} c_{w} f(w)-c_{e} f(e)+c_{e} \int_{C} f(t) d \omega_{C}(t) \\
& =\sum_{w \in D, w \neq e} c_{w} f(w)+c_{e} \int_{C} f(t) d \omega_{C}(t) \\
& =c_{e} \int_{C} f(t) d \omega_{C}(t),
\end{aligned}
$$

as $f$ vanishes on $D_{e}$. Since $f$ is non-constant on $C$, this integral is zero, by Theorem 5.

We conclude that

$$
\Delta_{m ; y_{1}, y_{2}, \ldots, y_{n+1}} * f(x)=0
$$

holds for each $x, y_{1}, y_{2}, \ldots, y_{n+1}$ in $C \cup D$, hence $f$ is a generalized $m$-exponential monomial on $C \vee D$ of degree at most $n$.

Now we assume that $i i$ ) holds, that is, $\left.f\right|_{C}$ is constant, and $\left.f\right|_{D}$ is a generalized $m_{D}$-exponential monomial of degree at most $n$ on $D$ with some exponential $m_{D}$ on $D$. Now we define $m: C \cup D \rightarrow \mathbb{C}$ as $m(x)=1$ for $x$ in $C$ and $m(x)=m_{D}(x)$ for $x$ in $D$. Then $m_{D}$ is an exponential on $C \vee D$, by Theorem 6. We claim that $f$ is a generalized $m$-exponential monomial of degree at most $n$ on $C \vee D$.

As $\left.f\right|_{C}$ is constant and $\left.m\right|_{C}=1$, we clearly have

$$
\Delta_{m ; y} * f(x)=0
$$

for each $x, y$ in $C$. It follows that

$$
\Delta_{m ; y_{1}, y_{2}, \ldots, y_{n+1}} * f(x)=0
$$

holds for each $x, y_{1}, y_{2}, \ldots, y_{n+1}$ in $C$. 
For $x$ in $C$ and $y$ in $D$ we have

$$
\Delta_{m ; y} * f(x)=f(x * y)-m(y) f(x)=f(y)-f(e) m_{D}(y)=\Delta_{m_{D} ; y} \cdot f(e) .
$$

By iteration, we have

$$
\Delta_{m ; y_{1}, y_{2}, \ldots, y_{n+1}} * f(x)=\Delta_{m_{D} ; y_{1}, y_{2}, \ldots, y_{n+1}} \cdot f(e)=0,
$$

for each $x$ in $C$ and $y_{1}, y_{2}, \ldots, y_{n+1}$ in $D$, as, by assumption, $\left.f\right|_{D}$ is a generalized $m_{D^{-}}$ exponential monomial of degree at most $n$ on $D$. We note that on the right side $\Delta$ is formed by using convolution in $D$.

For $x$ in $D$ and $y$ in $C$ we have

$$
\Delta_{m ; y} * f(x)=f(x * y)-m(y) f(x)=f(x)-f(x)=0,
$$

hence by iteration, we have again

$$
\Delta_{m ; y_{1}, y_{2}, \ldots, y_{n+1}} * f(x)=0
$$

whenever $x$ is in $D$ and there is a $y_{i}$ which is in $C$.

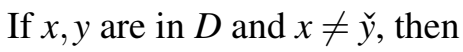

$$
\Delta_{m ; y} * f(x)=f(x * y)-m(y) f(x)=f(x \cdot y)-m_{D}(y) f(x) .
$$

On the other hand, if $x=\check{y}$, then

$$
\begin{aligned}
\Delta_{m ; \check{y}} * f(y) & =f(y * \check{y})-m_{D}(\check{y}) f(y) \\
& =f(y \cdot \check{y})+c_{e}\left(\int_{C} f(t) d \omega_{C}(t)-f(e)\right)-m_{D}(\check{y}) f(y) \\
& =f(y \cdot \check{y})-m_{D}(\check{y}) f(y),
\end{aligned}
$$

as $f(t)=f(e)$ for $t$ in $C$. By iteration, we conclude, that if $x, y_{1}, y_{2}, \ldots, y_{n+1}$ are in $D$ then

$$
\Delta_{m ; y_{1}, y_{2}, \ldots, y_{n+1}} * f(x)=\Delta_{m_{D} ; y_{1}, y_{2}, \ldots, y_{n+1}} \cdot f(x)=0,
$$

where $\Delta$ on the right side formed by using the convolution in $D$, and, by assumption, $\left.f\right|_{D}$ is a generalized $m_{D}$-exponential monomial of degree at most $n$ on $D$. The proof is complete.

\section{REFERENCES}

[1] W. R. Bloom and H. Heyer, Harmonic analysis of probability measures on hypergroups, ser. de Gruyter Studies in Mathematics. Walter de \& Co., Berlin, 1995. doi: 10.1515/9783110877595.

[2] Z. Fechner and L. Székelyhidi, "Sine functions on hypergroups," Arch. Math, vol. 106, no. 4, pp. 371-382, 2016, doi: 10.1007/s00013-016-0884-4.

[3] H. Heyer and S. Kawakami, "Extensions of Pontryagin hypergroups," Probab. Math. Statist., vol. 26, no. 2, pp. 245-260, 2006, doi: 10.1007/s00233-011-9322-x.

[4] Á. Orosz and L. Székelyhidi, "Moment functions on polynomial hypergroups in several variables," Publ. Math. Debrecen, vol. 65, no. 3-4, pp. 429-438, 2004.

[5] Á. Orosz and L. Székelyhidi, "Moment functions on polynomial hypergroups," Arch.Math. (Basel), vol. 85, no. 2, pp. 141-150, 2005, doi: 10.1007/s00013-005-1441-8. 
[6] Á. Orosz and L. Székelyhidi, "Moment functions on Sturm-Liouville hypergroups," Ann. Univ. Sci. Budapest. Sect. Comp., vol. 29, pp. 141-156, 2008.

[7] L. Székelyhidi, Functional Equations on Hypergroups. New Jersey, London: World Scientific Publishing Co. Pte. Ltd., 1995.

[8] L. Székelyhidi, "Functional equations on hypergroups," in Functional equations, inequalities and applications. Kluwer Acad. Publ., Dordrecht, 2003, pp. 167-181, doi: 10.1142/8481.

[9] L. Székelyhidi, "Spectral analysis and synthesis on polynomial hypergroups," Monatsh. Math, vol. 141, no. 1, pp. 33-43, 2004, doi: 10.1007/s00605-002-0003-4.

[10] L. Székelyhidi, "Exponential polynomials on commutative hypergroups," Arch. Math. (Basel), vol. 101, no. 4, pp. 341-347, 2013, doi: 10.1007/s00013-013-0559-3.

[11] L. Székelyhidi, "Characterization of exponential polynomials on commutative hypergroups," Ann. Funct. Anal., vol. 5, no. 2, pp. 53-60, 2014, doi: 10.15352/afa/1396833502.

[12] L. Székelyhidi, Harmonic and Spectral Analysis. New Jersey, London: World Scientific Publishing Co. Pte. Ltd., 2014. doi: 10.1142/8924.

[13] L. Székelyhidi and K. Vati, "Moment functions on hypergroup joins," submitted.

[14] L. Székelyhidi and K. Vati, "Functional equations on hypergroup joins," Arch. Math. (Basel), vol. 109, no. 1, pp. 41-47, 2017, doi: 10.1007/s00013-017-1022-7.

[15] M. Voit, "Substitution of open subhypergroups," Hokkaido Math. J., vol. 23, no. 1, pp. 143-183, 1994, doi: 10.14492/hokmj/1381412491.

[16] M. Voit, "Sine functions on compact commutative hypergroup," Arch. Math. (Basel), vol. 107, no. 3, pp. 259-263, 2016, doi: 10.1007/s00013-016-0918-y.

[17] R. Vrem, "Hypergroup joins and their dual objects," Pacific J. Math., vol. 111, no. 2, pp. 483-495, 1984, doi: 10.2140/pjm.1984.111.483.

Authors' addresses

Kedumetse Vati

Department of Mathematics, Shanghai Jiao Tong University, 800 Dongchuan Road, Minhang, China

E-mail address: kedumvati@yahoo.com

László Székelyhidi

Institute of Mathematics, University of Debrecen, H-4032 Egyetem tér, Debrecen, Hungary

E-mail address: lszekelyhidi@gmail.com 